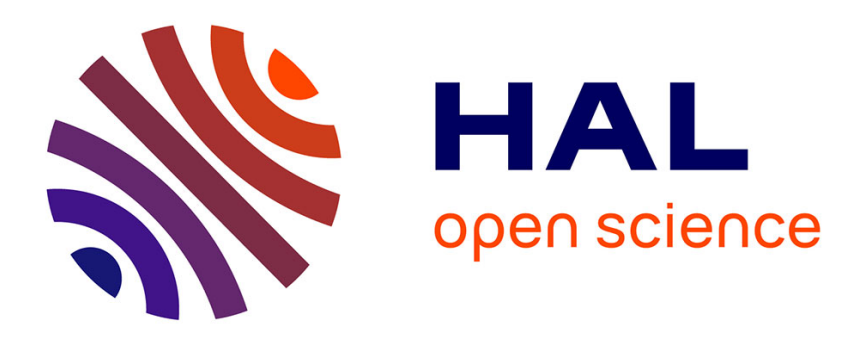

\title{
Particle in cell modelling of the observed modes of a dc wire discharge
}

R Gueroult, P Q Elias, D Packan, J Bonnet, Jean-Marcel Rax

\section{To cite this version:}

R Gueroult, P Q Elias, D Packan, J Bonnet, Jean-Marcel Rax. Particle in cell modelling of the observed modes of a dc wire discharge. Journal of Physics D: Applied Physics, 2010, 43 (36), pp.365204. 10.1088/0022-3727/43/36/365204 . hal-00569695

\section{HAL Id: hal-00569695 \\ https://hal.science/hal-00569695}

Submitted on 25 Feb 2011

HAL is a multi-disciplinary open access archive for the deposit and dissemination of scientific research documents, whether they are published or not. The documents may come from teaching and research institutions in France or abroad, or from public or private research centers.
L'archive ouverte pluridisciplinaire HAL, est destinée au dépôt et à la diffusion de documents scientifiques de niveau recherche, publiés ou non, émanant des établissements d'enseignement et de recherche français ou étrangers, des laboratoires publics ou privés. 


\title{
Particle in cell modelling of the observed modes of a DC wire discharge
}

\author{
R Gueroult ${ }^{1,2}$, P Q Elias ${ }^{1}$, D Packan ${ }^{1}$, J Bonnet $^{1}$ and J M \\ $\operatorname{Rax}^{2}$ \\ ${ }^{1}$ ONERA Palaiseau, 91761 Palaiseau, FRANCE \\ 2 LPP, Ecole Polytechnique, 91128 Palaiseau Cedex, FRANCE \\ E-mail: renaud.gueroult@polytechnique.edu
}

\begin{abstract}
Low pressure DC Wire Induced Plasma Sources (WIPS) exhibit two stable modes of discharge - constricted below a threshold pressure and diffuse above. Starting from experimental measurements, we conduct two dimensional particle in cell (PIC) modelling of a DC low pressure $\left(10^{-4}-10^{-2} \mathrm{mbar}\right)$, low current $(\sim 1 \mathrm{~mA})$ wire discharge in Helium. 2D PIC modelling is required to capture longitudinal non uniformity of the diffuse mode. PIC simulations reproduce the two discharge modes. Voltage versus pressure curve obtained from simulations matches fairly well experimental data, including the transition region. Discharge voltage dependence on pressure is analysed in consideration of electron impact ionization rates evolution with energy. In light of the PIC findings, a model of the discharge mode transition based on a Child Langmuir theory for ions is proposed. Confrontation with simulated data shows good agreement and validate the model for mode transition prediction. Simulations show that the diffuse mode is a space charge dominated regime.
\end{abstract}




\section{Introduction}

A Wire Induced Plasma Source (WIPS) is a type of low pressure gas discharge. Low pressure discharge specificity relies on the fact that the electron mean free path often exceeds the discharge chamber typical length scale, thus requiring a way of confinement. In the case of WIPSs, efficient confinement is achieved electrostatically [1], electrons being captured in the logarithmic potential generated between a cylindric cathode and a positively biased wire anode centered inside the cathode. This technique is the direct transposition - reversed polarity - of the one used in the Kingdon [2] ion trap, and permits low pressure $\left(\sim 10^{-5}\right.$ mbar $)$ gaseous discharge at very low applied voltage $(\sim 1 \mathrm{kV})$ within a chamber a few centimeters in size.

Consequently, WIPSs have been used as an ion sources for secondary emission electron guns [3], and their development have been pushed forward within this framework: versions of WIPS with multiple wires [4] and axial magnetic field [5] to increase their efficiency have been proposed. Nevertheless, those improvements are balanced by the increase weight and complexity of the system induced by the addition of magnets and/or wire tightening systems. Since then, whilst wire sources are widely used in electron guns, efforts to develop an understanding of the WIPS itself had been scarce. Most of the studies have been devoted to pulsed WIPS which can develop current density of about one Ampere per centimeter length, being therefore a good candidate as ion source for high current electron gun [3, 6, 7, 8]. Recently, Makarov undertook both an experimental [9] and a 1D numerical study [10, 11] of a high current pulsed WIPS and the temporal evolution of the discharge.

Experiments show that, depending on operating conditions, various modes of discharge are observed. This has been reported in various configurations: single [12] or multiple wires [13] at low pressure, single wire at higher pressure [14]. Low pressure DC WIPS in the milliAmpere range, used for example in electron beam fluorescence (EBF) optical diagnosis [15] electron guns, exhibits two distinct modes of discharge. Apart Pigache's [12] remark that, in the high pressure mode, the cathode sheath length was of the same order of the planar Child Langmuir length, to our knowledge, no thorough model of the DC low pressure, low current wire source was given. A better understanding of the main physical features of the wire discharge would benefit to EBF electron guns optimization and development of DC WIPS based technologies.

In this paper, we seek to examine the WIPS physics, and in particular its mode transition, in the light of an experimental characterization. We numerically investigate the operation of a DC low pressure $\left(10^{-4}-10^{-2}\right.$ mbar $)$, low current $(\sim 1 \mathrm{~mA})$ wire discharge which could typically be used in EBF electron guns. We limit our study to the single centered wire configuration without applied magnetic field. To begin with, an experimental characterization of the wire discharge is conducted in Section 2. Description of the specific features of our 2D particle in cell code follows in Section 3. PIC modelling validation is then achieved by confronting numerical results with experimental data in Section 4. Finally, based on simulations microscopic outputs we propose macroscopic models of observed phenomenon in Section 5. 


\section{Experimental characterization of the low pressure Wire Induced Plasma Source}

\subsection{Experimental Setup}

Experimental measurements have been made on the setup sketched in figure 1 . The cathode is made of a $R_{c}=2.5 \mathrm{~cm}$ inner radius, $L=20 \mathrm{~cm}$ length stainless steel cylinder. At one end of the cylinder, a silica window allows observation of the radial expansion of the discharge. Another window is installed laterally to monitor the longitudinal plasma development. The anode is a $R_{a}=0.1 \mathrm{~mm}$ radius Tungsten wire placed on the axis of the cylinder. Grounded end plates at each end provide longitudinal confinement. These disks have an inner hole diameter of $6 \mathrm{~mm}$.

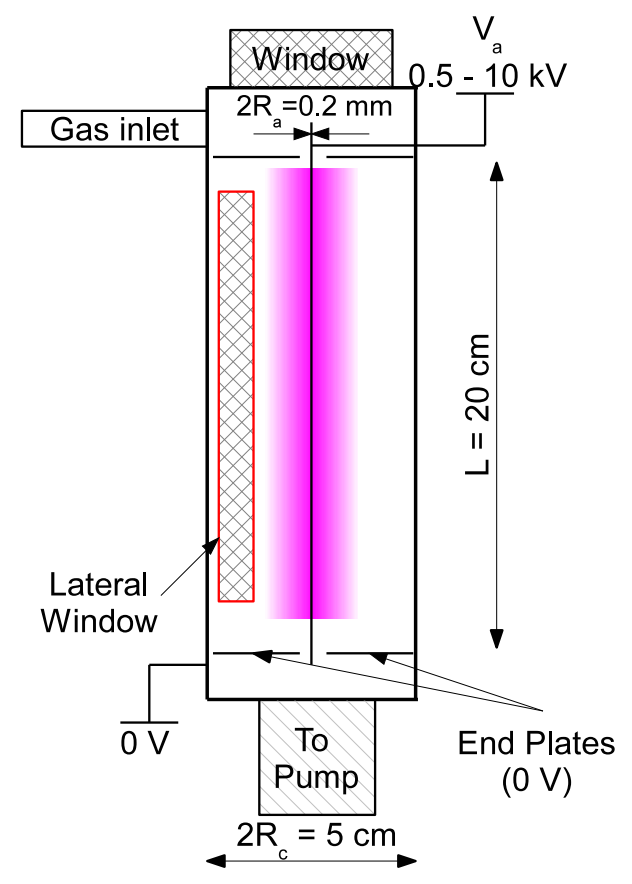

Figure 1. Experimental setup. The anode is a Tungsten wire $0.1 \mathrm{~mm}$ in radius, the cathode is a stainless steel cylinder $2.5 \mathrm{~cm}$ in radius and $20 \mathrm{~cm}$ long. The vessel is filled with Helium. Anode potential is $V_{a}=0.5-10 \mathrm{kV} \mathrm{DC}$. End plates assure longitudinal electrostatic confinement.

The plasma source was directly mounted on a Pfeiffer HiCube turbo pump reaching an ultimate pressure of $6 \cdot 10^{-7}$ mbar in measurement configuration. Measurements presented in this paper were done in Helium, but $\mathrm{Ne}, \mathrm{Ar}, \mathrm{Xe}, \mathrm{NO}$, $\mathrm{CO}, \mathrm{CO}_{2}$ and air have been successfully tested. Incoming gas flow was controlled by a an input valve. In order to have reliable reading of the pressure, several pressure gauges were compared and cross calibrated: two Baratron capacitive gauges, one hot 
cathode Bayard-Alpert gauge and one Pfeiffer Compact Full Range gauge. Pressure resolution in that configuration was about $10^{-5}$ mbar.

Typical anode voltage $\left(V_{a}\right)$ varies from $500 \mathrm{~V}$ to $10 \mathrm{kV}$ depending on the gas type and pressure. Heinzinger LNC stabilized DC power generators were used. A $+10 \mathrm{kV}$, $2 \mathrm{~mA}$ generator was used for low pressure measurements, whereas a $+1200 \mathrm{~V}, 10 \mathrm{~mA}$ generator was used for higher pressure.

Voltage and current were recorded using a P6015A Tektronix high voltage probe and a Hall current probe respectively, and displayed on a LeCroy $100 \mathrm{MHz}$ oscilloscope. Current was measured at the power generator output.

\subsection{Discharge Modes}

Two discharge modes are observed experimentally. These modes are similar to those described by Pigache [12] at higher currents. Figure 2 presents the discharge evolution with pressure.

At low values of pressure $\left(p \leq 10^{-2}\right.$ mbar), the plasma glow is limited to a region close to the anode, but fully expanded longitudinally. Therefore we refer to this mode as the constricted mode. When the pressure is decreased $\left(p<2.10^{-3} \mathrm{mbar}\right)$, the wire becomes red hot, and discharge voltage curve diverges. According to Francis [16], the divergence of the discharge voltage with pressure decrease is symptomatic of an obstructed discharge. This behaviour combined with, as it will be shown later, the potential profile near the anode, indicate that the constricted mode might be an obstructed discharge in a wire cylinder configuration.

On the other hand, when pressure is increased above the threshold value, the discharge switches to a diffuse mode. The transition is sudden, and a voltage drop of slightly less than a hundred Volts is observed. The system exhibits hysteresis on mode transition, the transition occurring at slightly lower (resp. higher) pressure when decreasing (resp. increasing) the pressure. In the diffuse mode, the plasma glow fills the whole radial inter electrodes volume but is limited longitudinally to a slice of a few centimeters . For a given pressure, the slice width increase with current, as shown in figure 3. In the diffuse mode, current increase is obtained at almost constant voltage, which is a characteristic feature of a normal glow discharge.

\section{Particle in cell modelling of low pressure discharge}

Longitudinal constriction of the discharge at the mode transition underlines the importance of $2 \mathrm{D}$ effects. It is therefore necessary to use a bi dimensional model to simulate the wire discharge behaviour. Moreover, Knudsen number well above one due to the low working gas pressure implies a particle in cell (PIC) numerical modelling.

In a classical manner $[17,18,19]$, a two dimensional $2 d 3 v(r, z)$ particle in cell code has been developed. Particles modelled are: electrons, ions and fast neutrals. Fast neutrals population is included mainly for secondary emission purposes. Indeed due to the high value of the charge exchange cross section in Helium, depending on pressure conditions, fast neutrals number density could be rather important and thus neutrals could play a role on secondary emission. Particles trajectories are computed using a Runge-Kutta 4th-order algorithm. The anode radius $R_{a}$ being order of magnitude smaller than the chamber radius $R_{c}$, a non-uniform cylindrical mesh has been used to minimize the number of cells. Nevertheless, it is strictly necessary to resolve properly 


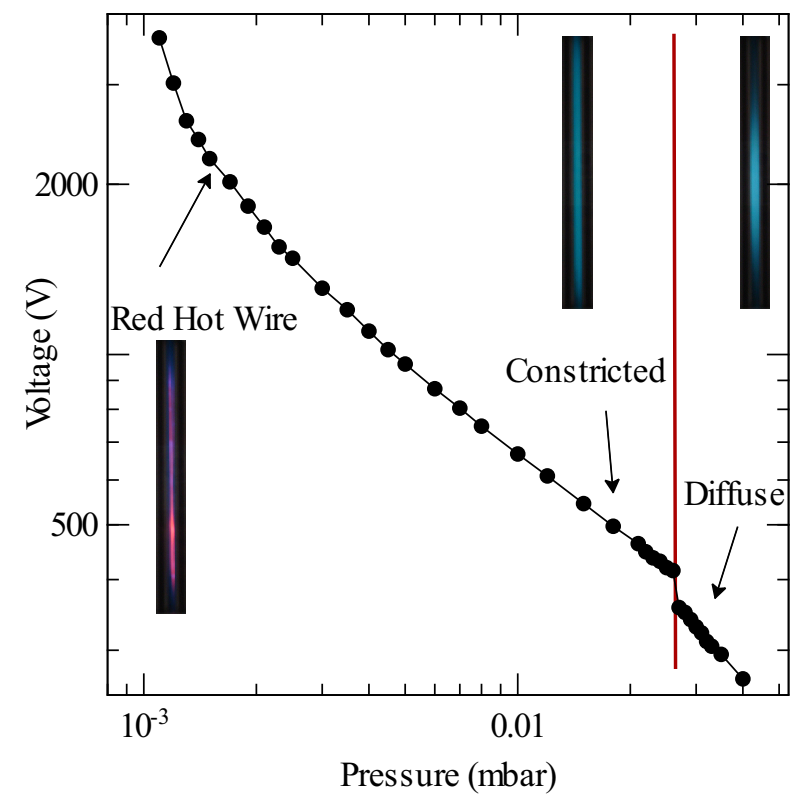

Figure 2. Voltage vs pressure curve for a $1 \mathrm{~mA}$ current. The diffuse mode is observed at high pressure, transition (red line) occurs when decreasing pressure and the constricted mode takes place. At lower pressure, the anode becomes red hot.

the wire to compute high field gradients in this area and hence to integrate properly the particles trajectories.

Despite the low working pressure, collisions are fundamental to model the discharge. Consequently, we implemented a complete Monte Carlo Collision (MCC) module [19]. Collisions taken into account in the simulation are synthesized in table 1. The corresponding cross sections are those gathered by Hartmann [20], exception made of electron impact excitation for which we used Stone's [21] data. Indeed, Hartman [20] cross sections for this process differ significantly from standards [21]. Regarding excitation process, only one excited level is taken into account, but the energy loss during the excitation process is computed randomly between the energy of the first excited level and the ionization energy. For a given collision process, energy distribution between all particles involved is computed by inverting the differential cross section $\sigma(E, \chi)$ for that collision [19, 22]. Gas dependent fitting parameters for ionization processes are taken from Opal [23]. Null collision technique [24] has been implemented to speed up calculations.

Due to the low working pressure, collisions between active populations are neglected. Similarly, the low ionization fraction justifies the implementation of a fixed density background gas.

Secondary emission models are used for all three species impacts on the electrodes. The processes involved for heavy particles are kinetic emission (ions, fast neutrals) and potential emission (ions) [25, 26]. For ion impact secondary electron emission at a few hundreds electron volts, fitting parameters references in the literature are scarce. For example, Bohm [27] gives a value $\gamma_{\mathrm{He}^{+}} \sim 0.25$ emitted electrons per incident ion at 


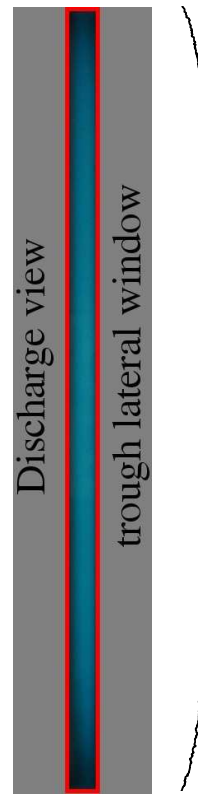

(a)

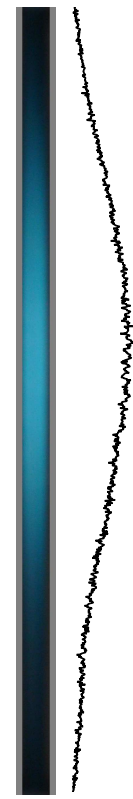

(b)

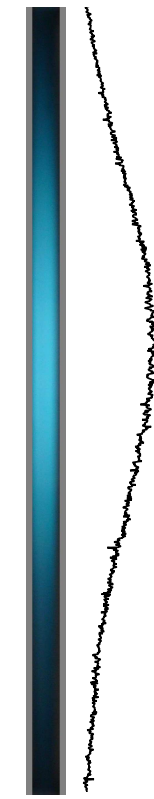

(c)

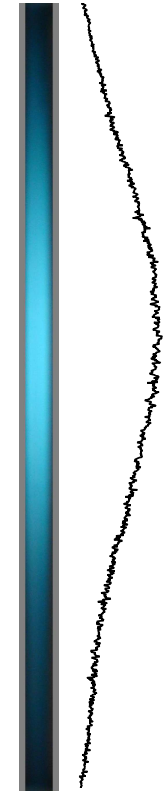

(d)

Figure 3. Discharge profiles views trough the lateral window - 3(a): Constricted mode $p=1.10^{-2}$ mbar, $I=1 \mathrm{~mA}-3(\mathrm{~b}), 3(\mathrm{c}), 3(\mathrm{~d})$ Diffuse mode $(p=$ 2.8.10 ${ }^{-2}$ mbar) longitudinal expansion with current for $I=1,1.5$ and $2 \mathrm{~mA}$.

\begin{tabular}{ccc|c}
\hline \multicolumn{4}{c}{ Electron impact induced processes } \\
\hline $\mathrm{e}^{-}+\mathrm{He}$ & $\rightarrow$ & $\mathrm{e}^{-}+\mathrm{He}$ & Elastic \\
$\mathrm{e}^{-}+\mathrm{He}$ & $\rightarrow$ & $\mathrm{e}^{-}+\mathrm{He}^{\star}$ & Excitation \\
$\mathrm{e}^{-}+\mathrm{He}$ & $\rightarrow$ & $2 \mathrm{e}^{-}+\mathrm{He}^{+}$ & Ionisation \\
\hline \multicolumn{4}{c}{ Ion impact induced processes } \\
\hline $\mathrm{He}^{+}+\mathrm{He}$ & $\rightarrow$ & $\mathrm{He}^{+}+\mathrm{He}$ & Elastic \\
$\mathrm{He}^{+}+\mathrm{He}$ & $\rightarrow$ & $\mathrm{He}+\mathrm{He}^{+}$ & Charge Exchange \\
$\mathrm{He}^{+}+\mathrm{He}$ & $\rightarrow$ & $\mathrm{He}^{+}+\mathrm{He}^{\star}$ & Excitation \\
$\mathrm{He}^{+}+\mathrm{He}$ & $\rightarrow$ & $2 \mathrm{He}^{+}+\mathrm{e}^{-}$ & Ionization \\
\hline \multicolumn{4}{r}{ Fast neutral impact induced } \\
\hline $\mathrm{He}^{f}+\mathrm{He}$ & $\rightarrow$ & $\mathrm{He}^{f}+\mathrm{He}$ & processes \\
$\mathrm{He}^{f}+\mathrm{He}$ & $\rightarrow$ & $\mathrm{He}^{f}+\mathrm{He}$ & Elastic \\
$\mathrm{He}^{f}+\mathrm{He}$ & $\rightarrow$ & $\mathrm{He}^{f}+\mathrm{He}^{+}+\mathrm{e}^{-}$ & Ionitation \\
\hline
\end{tabular}

Table 1. Active Species - Background gas collision processes modeled in the simulations. Corresponding cross section are from Hartmann[20], except for electron impact excitation taken from Stone [21].

$350 \mathrm{eV}$ whereas Szapiro [28] has $\gamma_{\mathrm{He}^{+}} \sim 0.6$ at $500 \mathrm{eV}$, both for clean stainless steel. In addition, the secondary emission coefficient depends strongly on surface condition, this parameter being hardly known. Following Bohm [27], simulations are made using

$$
\gamma_{H e^{+}}=0.3, \quad \gamma_{H e^{0}}=0.1,
$$


as default values. Influence of those parameters will be analysed parametrically. Impacting electrons can be elastically or inelastically reflected, or create true secondaries [29, 30, 31].

Depending on the plasma impedance, ideal voltage sources can induce instabilities. It is thus desirable to drive the plasma with a ideal current source. The anode potential evolves due to the cumulated effect of impacting particles and electrons drawn by the current source. Simulation runs until electrodes current have converged to the specified value.

\section{Code validation: comparison with experiments}

In order to validate the particle in cell code described in the previous section, test cases are run and results are confronted to experimental data. Simulations results are compared with experiments on two different levels. First visual aspect and spatial properties of the simulated discharge are compared qualitatively to experimental pictures. Then computed voltage versus pressure curve is examined in light of the experimental one.

\subsection{Discharge Modes: spatial properties}

Three cases matching the experimental modes of discharge are numerically modelled. Working conditions of these cases are summarized in table 2. The first one corresponds to a constricted mode at a $1 \mathrm{~mA}$ discharge current (Case A). The others are diffuse modes for 1 and $2 \mathrm{~mA}$ (Cases $\mathrm{B}$ and $\mathrm{C}$ respectively).

\begin{tabular}{cccc}
\hline & Case A & Case B & Case C \\
\hline Mode & Constricted & Diffuse & Diffuse \\
Pressure $\left(10^{-2}\right.$ mbar $)$ & 1.36 & 6.07 & 6.07 \\
Current $(\mathrm{mA})$ & 1 & 1 & 2 \\
\hline
\end{tabular}

Table 2. Simulations conditions used for comparison with experimental discharge modes

Figure 4 presents the Abel transform of the volumic excitation rate for the three cases. The Abel transform of the excitation rate is directly related to the emitted light and its comparison with observed glow is a good indicator. Simulations confirm the existence of a constricted mode of discharge where excitation is localized near the anode and uniform longitudinally. At higher pressure, for the same $1 \mathrm{~mA}$ discharge current, the excitation area increases radially and collapses longitudinally. The longitudinal expansion with current is well modelled by the simulations.

Longitudinal profiles of the Abel transform of excitation are plotted with profiles obtained from experimental pictures in figure 5 . No correction has been made to the raw data, except a normalization of all simulated curves with a single coefficient equal to the ratio of the observed maxima over the simulated one for the $2 \mathrm{~mA}$ diffuse mode case. Relative levels of intensity for simulated and experimental data are almost equal, and shapes, although not exactly superimposed, are qualitatively identical. Indeed, simulated emission profiles maxima are more peaked than experimental ones. Reflected light - not modelled in our PIC code - is thought to contribute to the peak broadening. Indeed excitation produces photons with directions distributed equally on 


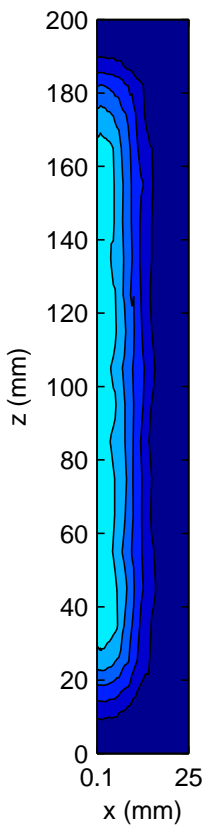

(a)

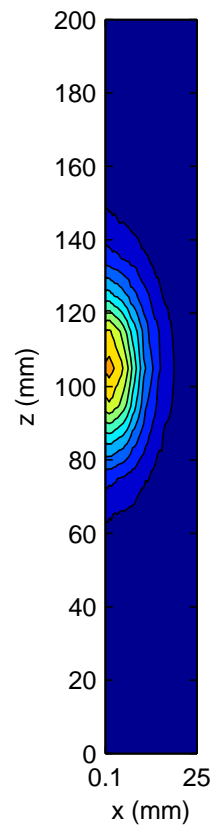

(b)

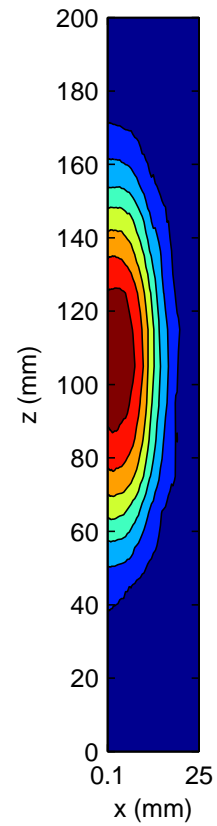

(c)

Figure 4. Simulated discharge modes: 4(a) Constricted mode at $1 \mathrm{~mA}$ discharge current, $p=1.3610^{-2} \operatorname{mbar}$ (case A)- 4(b) and 4(c) Diffuse mode at $p=$ $6.0710^{-2}$ mbar for 1 and $2 \mathrm{~mA}$ discharge current respectively (cases $\mathrm{B}$ and $\mathrm{C}$ )

a solid sphere angle, and only a small fraction of it hit directly the camera detector. Another fraction of photons is diffused from the cathode surface and then possibly enter the camera detector. Diffusion can take place in areas far from the median plane, increasing in turn the signal on both sides of the excitation maximum.

\subsection{Discharge voltage variation with pressure.}

The simulated discharge voltage versus pressure is plotted along with experimental data in figure 6 . The sharp transition observed experimentally between the two modes is found as well on the simulated curve. The voltage jump is well defined, and curves evolutions around the mode transition are very similar: computed curves slopes above and below the mode transition match closely the experimental ones. Discrepancy between simulated and experimental data is mainly present on the high pressure region, where, as it will be shown in the next paragraph, secondary emission coefficient value play a role. For lower pressures, particle in cell results stick closely to the experimental ones, modelling properly the strong rise of discharge voltage with pressure decrease.

\subsection{Influence of secondary emission coefficients}

As mentioned in Section 3, constant secondary electron emission value for ion and fast neutral impact were used due to the lack of data in the literature. A parametric 


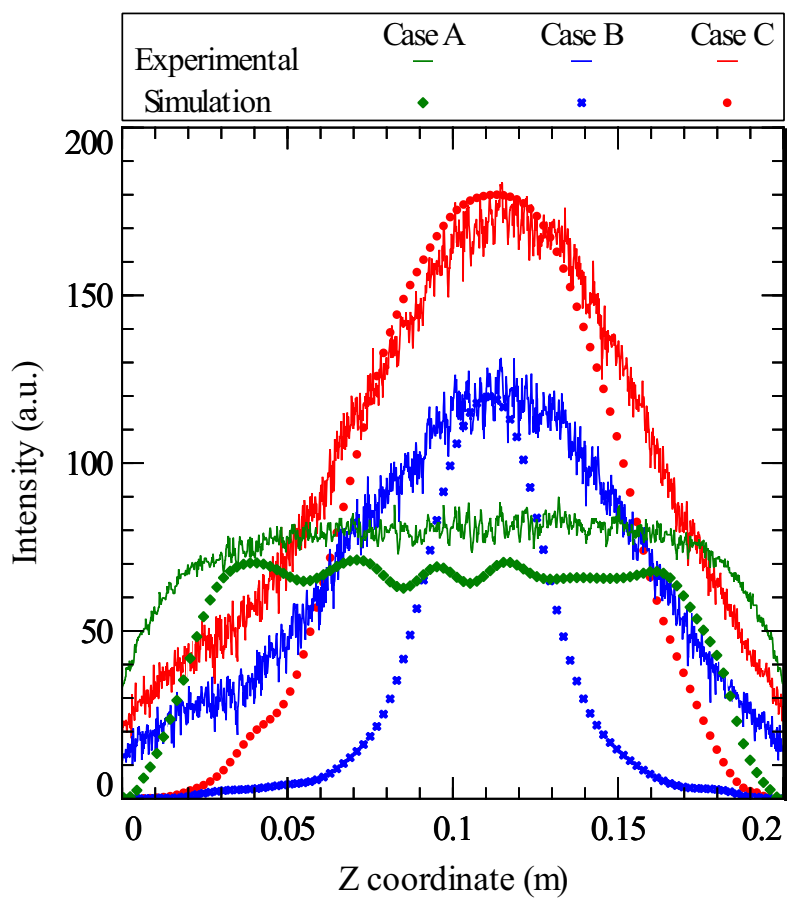

Figure 5. Simulated and experimental plasma longitudinal expansion profiles. Simulations conditions are summarized in table 2.

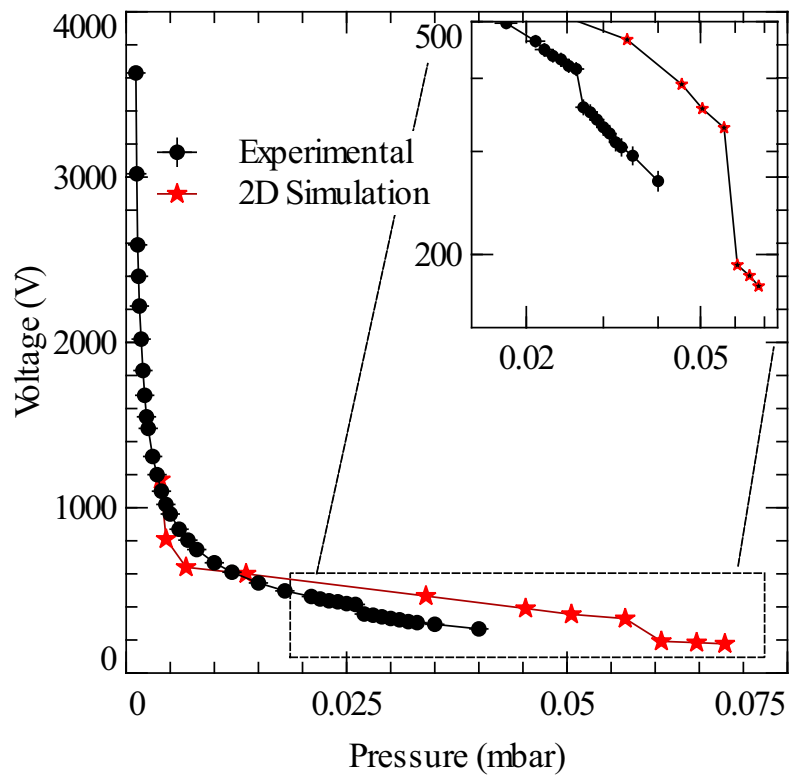

Figure 6. Experimental and simulated discharge voltage versus pressure for $1 \mathrm{~mA}$ current. Voltage jump of slightly less than a hundred volts is observed on both curves. 
study was made to discriminate the influence of these coefficients on the simulations. Before any computational study, an analysis could be formulated as follows. Increasing secondary emission coefficients would lead to higher electron population number $N_{e^{-}}$, and in turn to higher ionization rate, yielding eventually higher discharge current $\left(I>I_{0}\right)$. On the pressure range considered, the plasma is resistive, meaning that the original $I_{0}$ discharge current can be obtained at lower voltage using higher value of $\gamma$. Moreover, the fraction of secondary electrons returning to the cathode increases with mean free path, thus dependence on $\gamma$ should diminish with pressure decrease. Simulations made with $\gamma_{\mathrm{He}^{+}}=0.6$ validates this analysis: the transition occurs numerically at the same voltage $V \sim 330 \mathrm{~V}$ despite of a drop of the mode transition pressure from $p_{t}=6.10^{-2}$ mbar for default $\gamma_{H e^{+}}$value to $p_{t}=4.4 .10^{-2}$ mbar. Influence of secondary emission on the voltage versus pressure curve given in figure 6 is hence mainly a shift to the left, the mode transition occurring then at lower pressure. This shift will be explained by the mode transition model proposed in Section 5 .

To summarize, secondary emission values tuning could diminish, if not remove, discrepancies between simulations and experimental data in terms of pressure of transition. Considering that secondary emission values do not change the fundamental behaviour - mode transition, spatial properties of the modes - of the discharge on the pressure range considered and that these coefficients are hardly predictable, no further attention has been paid to this phenomena.

\section{Discussion}

Numerical results on the previous test cases justify the use of the code as a mean to dig out microscopic data, e.g. ionization volumic rates or densities, to propose macroscopic model of the discharge.

\subsection{Model of the mode transition}

Based on experimental measurements, Pigache [12] noted that the high pressure cathode sheath width was of the same order as the Child Langmuir length computed for an Helium ion current between planar electrodes. Makarov [9] used the same approach to describe one state of its pulsed wire discharge. Those analyses rely on the hypothesis that the discharge can be on first approximation considered as an unipolar current of Helium ions. In order to extend Pigache's argument to the mode transition analysis, this hypothesis has to be justified at first.

When pressure is increased, the ion mean velocity decreases because of the lower discharge voltage and higher collision rate with the neutral background gas. Considering that the cathode current is mainly an ion current, in order to sustain a given current, the ion population number should increase to balance the velocity decrease. This way, the ion flux to the cathode remains constant. Due to charge exchange collisions, fast neutrals population follows the ion population increase, until pressure reaches the threshold value. Beyond this limit, the fast neutrals energy is limited as well by a lower discharge voltage and elastic collisions, and hence more and more fast neutrals are transferred back to the background gas population as pressure increases. Indeed neutrals are monitored as fast neutrals as long as their energy exceeds a given value, taken as a threshold for kinetic secondary emission on electrodes.

Simulations results presented in figure 7 confirms these variations. The ion population number is about ten times more important than electron one's in the 
vicinity of the mode transition. Charged particles are thus mainly ions, justifying the study of the discharge as a space charge limited ion current between electrodes. Nevertheless, ion neutral cross sections for elastic and charge exchange processes in Helium $\left(\sigma \sim 2.10^{-19} \mathrm{~m}^{2}\right)$ indicate that, at the pressure of transition, a typical ion has approximately five collisions on its way to the cathode. Collisions limit ion speed, increasing space charge and in turn limiting the sustainable current. Consequently, accounting for collisions is important to model the transition.

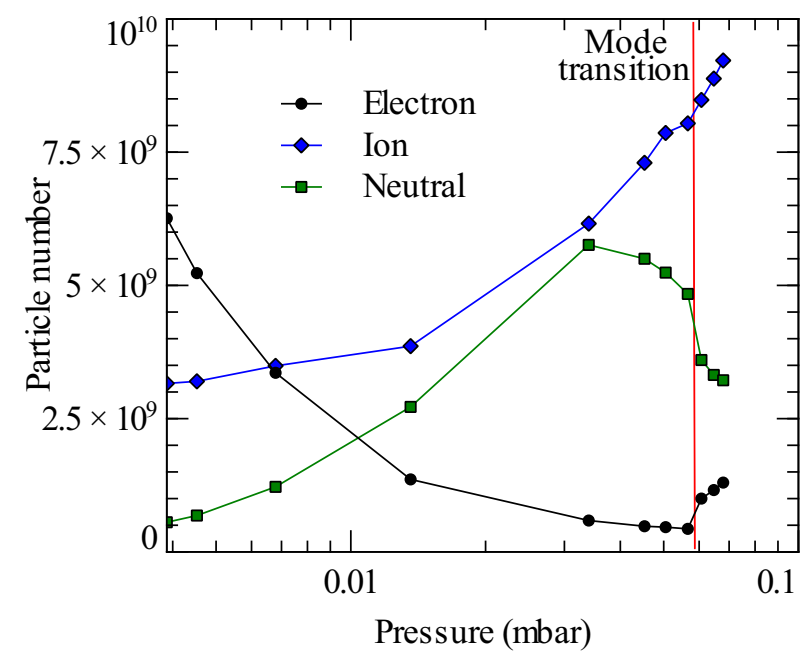

Figure 7. Evolution of electrons, ions and fast neutrals population numbers with pressure. Plasma is mainly made of ions at high pressure, whereas electrons number increase strongly at lower pressures.

Thus, assuming an ion current between cylindrical electrodes, and using the collision frequency to model ion impacts, the Child Langmuir problem can be stated as

$$
\begin{aligned}
& \frac{d}{d r}\left(r \frac{d V}{d r}\right)=-\frac{I}{2 \pi \epsilon_{0} L v}, \\
& m v \frac{d v}{d r}=-e \frac{d V}{d r}-\nu m v,
\end{aligned}
$$

(2) and (3) being the Poisson's equation expressed in cylindrical coordinates and the momentum equation for helium ions, respectively. Here $V$ is the electric potential, $v$ is the ion speed, $I$ is the discharge current, $L$ is the discharge length, $m$ is the Helium ion mass, $e$ is the elementary charge and $\nu=\sigma n_{g} v$ is the ion collision frequency. Resolving numerically the system formed by (2) and (3) for ions a having thermal velocity at the anode with

$$
\begin{aligned}
& V\left(R_{a}\right)=V_{a} \\
& V\left(R_{c}\right)=0
\end{aligned} \quad E\left(R_{a}\right)=-\frac{d V}{d r}\left(R_{a}\right)=0
$$

yields the Child Langmuir limiting current $I_{C L}$ for the studied configuration - anode potential and electrodes geometry. $I_{C L}$ is obtained when the space charge cancels the electric field at the anode, meaning that no more ions could be drawn. 
For comparison, in the analytic situation of a classical Child Langmuir current between planar electrodes [32], $I_{C L}$ is expressed as

$$
I_{C L_{\|}} \propto \frac{V^{3 / 2}}{d^{2}}
$$

where $d$ is the inter electrode gap and $\mathrm{V}$ the applied voltage. In our situation, the Child Langmuir limiting current is a function of $V$, the inter electrode gap $\delta R$, as well as the pressure $p$ due to the collisional term in (3):

$$
I_{C L}=I_{C L}(V, \delta R, p) \text {. }
$$

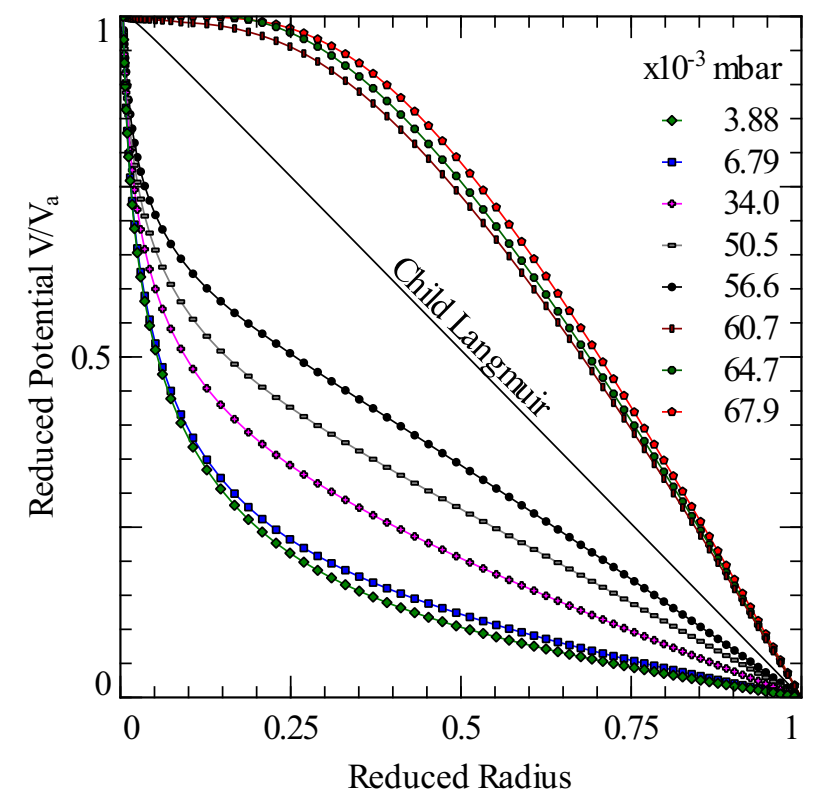

Figure 8. Child Langmuir limit for the pressure of mode transition and PIC simulations reduced potential $V / V_{A}$ at $z=z_{m}$ against reduced radius $r / R_{c}$ for various gas pressures. When increasing pressure, the positive space charge builds up near the anode. Above a given pressure, the voltage profile crosses the one obtain from Child Langmuir model and transition occurs. Potential is then flattened near the anode to sustain higher currents.

Remembering that the discharge voltage decreases with pressure, the discharge mode transition for a given current $I_{0}$ can be explained as follows. Figure 8 shows the reduced potential $V / V_{a}$ versus reduced radius $\left(r / R_{c}\right)$ at $z_{m}=L / 2$ on a wide range of pressure values obtained from PIC simulations, as well as the Child Langmuir limit obtained using the pressure $p=p_{t}$ of mode transition in the collisional term. At low pressure, the discharge voltage is high enough compared to the inter electrode gap $\delta R$ to collect $I_{0}<I_{C L}\left(V, \delta R, p_{t}\right)$. When increasing pressure, thus decreasing voltage, the discharge voltage reaches $V_{t}$, the computed Child Langmuir potential for $I_{0}$ and $\delta R$, so that $I_{0}=I_{C L}\left(V_{t}, \delta R, p_{t}\right)$. Above this pressure, it is no longer possible to sustain the discharge current $I_{0}$ without reducing the sheath width. Transition occurs and a space charge builds up near the anode. Grounded end plates push electrons away, favouring space charge build up close to the longitudinal mid plane. This space charge 
flatten the radial potential profile near the anode, in turn decreasing the sheath width and hence permitting stronger current. Flat behaviour of the potential at $z \sim z_{m}$ near the anode observed in figure 9 creates a longitudinal confinement of electrons near the mid plane. Electrons therefore oscillates longitudinally close to $z_{m}$, increasing in turn the number density and confinement in that area. The plasma is no longer uniform longitudinally, but centered near the mid plane defined by $z=z_{m}$. This longitudinal constriction justifies the fact that diffuse mode sheath width has a minimum. Indeed, working at constant current $I=2 \pi r L j(r)$, a longitudinal collapse induces an increase of $j(r)$. To sustain this current density rise without increasing the potential drop across the sheath, a step decrease of the sheath width is necessary.

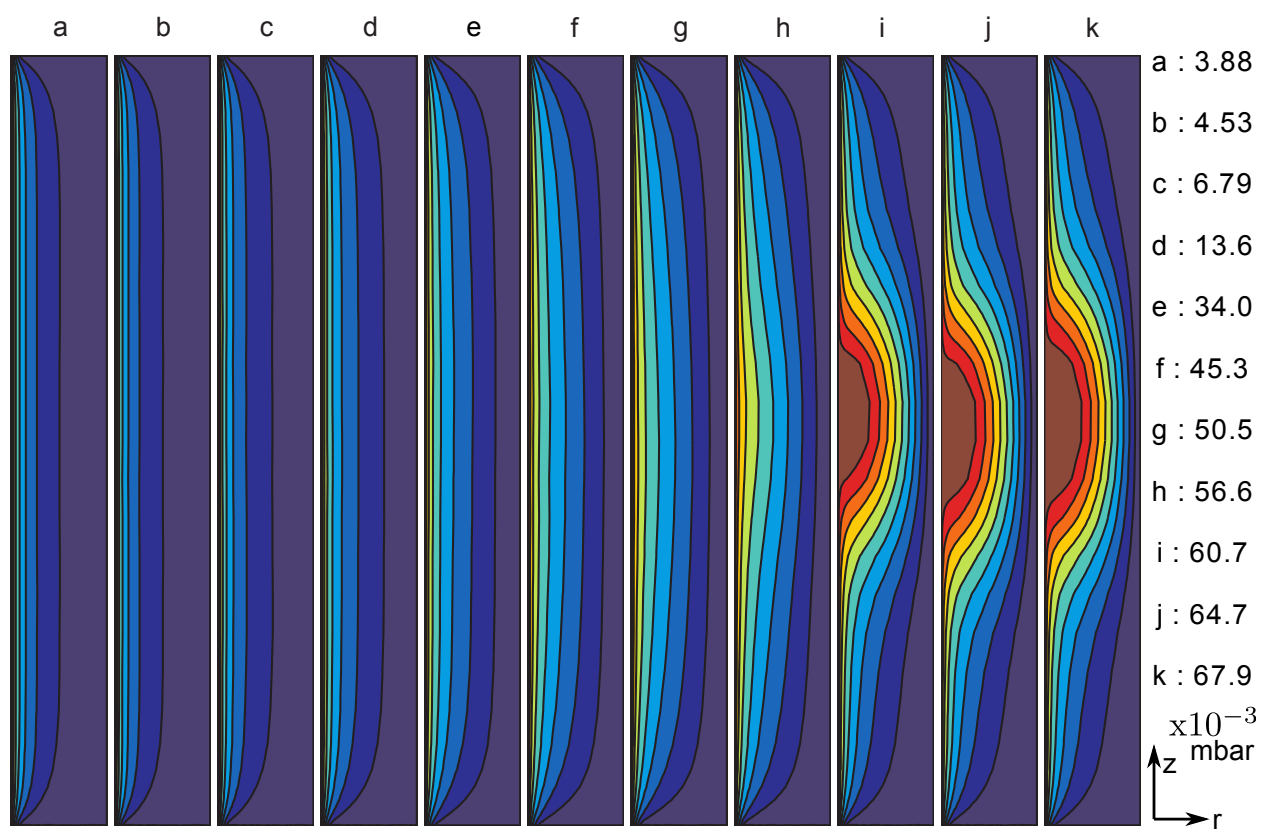

Figure 9. Evolution of the normalized electric potential distribution in the $(r, z)$ plane with pressure. The formation of the electron confinement area existing in the diffuse mode is clearly seen.

Using this modified Child Langmuir model, it is possible to predict the voltage of the mode transition for a given electrodes geometry and discharge current. Moreover, knowing the voltage drop across the transition, we can calculate the sheath width of the diffuse mode and reciprocally. Results obtained from experimental measurements, simulations and Child Langmuir model are compared in table 3. Data show fair agreements and confirm the efficiency of the modified Child Langmuir model for mode transition prediction.

To summarize, the mode transition is the result of a positive space charge growth beyond the threshold value, inducing a space charge limited regime. Transition is observed when current exceeds the Child Langmuir current computed for the given configuration. The decrease of the mode transition pressure $p_{t}$ noted in Section 4 is the direct consequence of the accelerated build up of positive space charge, higher value of secondary emission coefficient inducing higher electron number density $n_{e}$. 


\begin{tabular}{cccc}
\hline & Experimental & Child Langmuir model & Simulation \\
\hline Transition voltage $(\mathrm{V})$ & 415 & 320 & 330 \\
Sheath width $(\mathrm{mm})$ & - & 12 & 14 \\
\hline
\end{tabular}

Table 3. Comparative of experimental, simulation and Child Langmuir model results for mode transition prediction

Indeed, $n_{e}$ being many times lower than the ion number density $n_{i}$ near the mode transition, a small increase of $n_{e}$ will induce a larger increase of $n_{i}$, and consequently the positive space charge limit for mode transition will be obtained at lower pressure.

Phenomenologically speaking, potential profiles given in Figure 8 provide a new feature differencing the constricted and diffuse modes: the existence of an electric field at the anode. Following Donko's [33] proposition of definition of an obstructed discharge -the electric field does not vanish at the anode, the constricted mode could be an obstructed discharge mode between coaxial cylinders.

\subsection{Discharge voltage dependence on pressure}

For WIPS based technologies, working conditions should exclude regions of $V-p$ curves divergence. Indeed divergence of the $V-p$ curve at a given current requires higher power input, which is to be avoided as much as possible. It is therefore important to get insights into the discharge voltage behaviour.

The wire cylinder configuration permits low pressure gaseous discharge at low discharge voltage. Indeed, although $V-p$ curves look like typical Paschen's curves, discharge can be operated at pressure orders of magnitude lower that what would predict Paschen's law for a parallel plates configuration and a given potential. Even though the generalisation of Paschen's law to non uniform electric field between coaxial cylinders [34, 35] induces slight modifications, it can not explain the low voltage ignition. Actually, due to the small anode radius used in our configuration, a large fraction of electrons misses the anode and orbits around it. The effective electron path for ionization is therefore much longer than the distance an electron would travel if it falls directly on the anode (hypothesis implicitly used for the derivation of Paschen's law between coaxial cylinders). The direct consequence of this longer electron path is the capability to satisfy the breakdown criterion at much lower pressure, making it possible to operate discharges at $1 \mathrm{kV}$ for pressure orders of magnitude lower that what would predict Paschen's law [34]. To summarize, the observed deviation from Paschen's law results from the use of a very small anode.

The study of the $V-p$ curve divergence relies on the electron population number $N_{e}$ balance in the discharge. Considering that the anode current $I$ is an electron current, the electron population number conservation can be expressed as:

$$
\frac{d N_{e}}{d t}=n_{g} \sum_{j=1}^{N_{e}} \zeta_{e^{-}}^{j}-I / e
$$

where $\zeta_{e^{-}}^{j}=\sigma_{e^{-}}^{i}\left(v_{e^{-}}^{j}\right) v_{e^{-j}}$ is the reduced ionization frequency - ionization collision rate over number density - for the $j^{\text {th }}$ electron, $N_{e}$ is the total number of electrons in the discharge, $v_{e^{-}}^{j}$ is the speed of the $j^{t h}$ electron, $\sigma_{e^{-}}^{i}$ is the ionization cross section for electron impact and $n_{g}$ is the neutral gas density. Secondary emission of electrons from ions and fast neutrals impact on cathode should have been included in (7). 
Nevertheless, our interest here is in the low pressure range where secondary electrons are mainly impacting back on the cathode after crossing the discharge chamber without collisions. We thus neglect both secondary emission of electrons and electron current at the cathode, which are supposed to balance each other. Ion and fast neutral impact ionization phenomenon, which are found to be of second order, have been neglected as well.

Introducing the mean reduced electron ionization rate $\zeta_{m}$ and the anode electron collection rate $\nu_{a}$ as

$$
\zeta_{m}=\frac{1}{N_{e}} \sum_{j=1}^{N_{e}} \zeta_{e^{-}}^{j}
$$

and

$$
I=e N_{e} \nu_{a}
$$

equation (7) becomes

$$
\frac{d N_{e}}{d t}=N_{e}\left(n_{g} \zeta_{m}-\nu_{a}\right) .
$$

At steady state, $n_{g} \zeta_{m}=\nu_{a}$. In addition, anode current $I$ and set point current $I_{0}$ have to be equal, so $n_{g} \zeta_{m}=\left.\nu_{a}\right|_{I=I_{0}}=\nu_{a_{0}}$. Hence, a pressure decrease has to be compensated by an increase of $\zeta_{m}$ or a decrease of $\nu_{a_{0}}$.

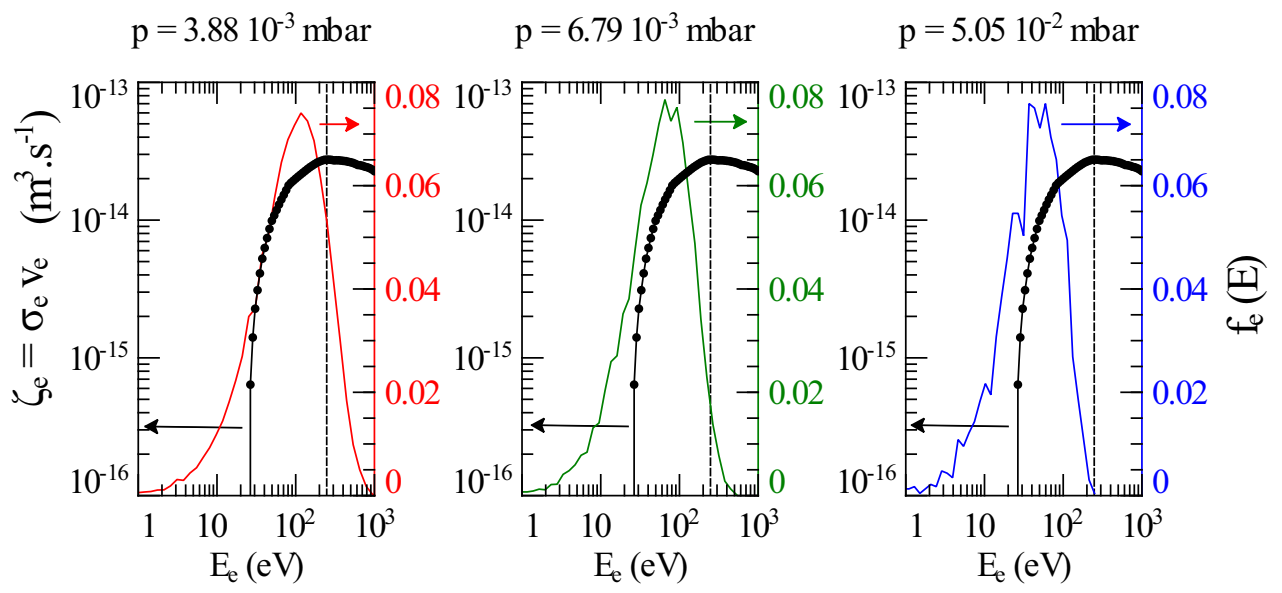

Figure 10. Electron energy distribution function EEDF is plotted for different pressure along with the reduced electron ionization impact frequency $\zeta_{e^{-}}=$ $\sigma_{e^{-}}^{i}\left(v_{e^{-}}\right) v_{e^{-}}$obtained from cross section.

As shown in figure $10, d \zeta_{e^{-}} / d E_{e}$ decreases with electron energy $E_{e}$, becoming negative above threshold value $E_{e_{T}}$ - energy for which $\zeta_{e^{-}}$is maximum. Therefore, for an individual electron, increasing $\zeta_{e^{-}}$by $\Delta \zeta_{e^{-}}$requires a larger $\Delta E_{e}$ as $E_{e}$ increases, until it gets impossible $\left(E_{e} \geq E_{e_{T}}\right)$. Generalizing to the whole electron population, $\zeta_{m}$ has a qualitatively similar behaviour, exhibiting a maximum as the electron energy distribution functions (EEDF) peaks around $E_{e_{T}}$. As featured in figure 11, electron mean energy varies almost linearly with the discharge voltage in the constricted mode. Consequently, behaviour of $\zeta_{m}$ as function of electron mean energy or discharge voltage 
is identical. This behaviour is well illustrated by the EEDF obtained from PIC simulations (see figure 10). At higher pressure, electrons temperature is low and a voltage increase results in the increase of the ionization cross section of all electrons, leading to a large increase of $\zeta_{m}$. When pressure decreases, due to the combined effect of a lower slope of $\zeta_{e^{-}}\left(E_{e}\right)$ and an increase of the fraction of electrons having an energy above $E_{e_{T}}, \partial \zeta_{m} / d V$ decreases. Decreasing further the pressure will lead $\zeta_{m}$ to reach a maximum and then to decrease.

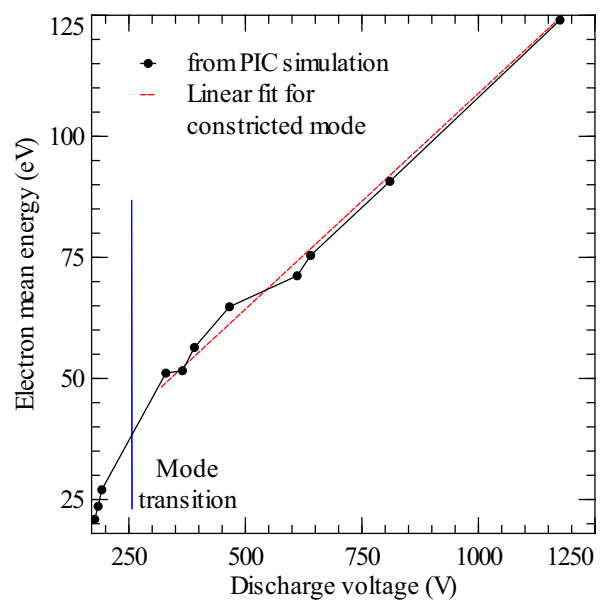

Figure 11. Electron mean energy versus discharge voltage obtained from PIC simulations. Data corresponding to the constricted mode are well fitted by a linear curve (red dashed curve).

On the other hand, $\nu_{a_{0}}$ can be seen as a measure of the electron confinement. The lower $\nu_{a_{0}}$, the better is the confinement. On the lower voltage range, $\nu_{a_{0}}$ remains almost constant as low primary electron energy leads mainly to isotropic scattering. As voltage increases, higher electron mean temperature leads to a smaller angle of scattering and higher energy for secondary electrons. Smaller scattering angles allow primaries to keep on their path and miss the anode. Higher energy for secondaries increases their chance to miss the anode. Hence $\nu_{a_{0}}$ is a decreasing function of the electron mean energy and, in turn, of voltage. Scattering models [19] shows that the higher the electron energy $E_{e}$, the higher is the $\Delta E_{e}$ required to decrease the angle of scattering or increase the energy of secondaries by a given value. Therefore, after passing through a maximum, $\left|\partial \nu_{a_{0}} / \partial V\right|$ decreases with electron energy or anode potential.

Evolution of $\zeta_{m}, \nu_{a}$ and $N_{e}$ with a diminution of pressure is obtained from time constants of the various processes involved. As pressure is decreased, the anode current $I$ and thus $\nu_{a}$ diminish in a fraction of $\tau=\left(n_{g} \zeta_{m}\right)^{-1}$ - the inverse of the ionization rate. Then, as $I$ is lower than set point value $I_{0}$, a positive charge builds up on the anode and, in turn, the anode potential rises as $\left(I_{0}-I\right) t$ until $I$ return to $I_{0}$. As anode potential increases, $\zeta_{m}$ evolves in about the time it takes for an electron to make one orbit around the wire. This period is about $10^{-8} \mathrm{~s}$ for an electron of a few hundreds of $\mathrm{eV}$, being smaller than $\tau$ which varies from $8 \cdot 10^{-8}$ to $7 \cdot 10^{-7} \mathrm{~s}$ depending on pressure. Equation (7) shows that $N_{e}$ evolution is slower, with a time constant of a few $\tau$.

The evolution of $\partial V / \partial p$ can thus be summarized as follows. At higher pressure, 
the voltage increase $\Delta V$ needed to get a sufficient growth of $\zeta_{m}$ to balance the $n_{g}$ decrease is small, and thus obtained quickly. Therefore $\left(n_{g} \zeta_{m}-\nu_{a}\right)$ returns to zero before $N_{e}$ had time to evolve. Besides, small $\Delta V$ at low discharge voltage leaves $\nu_{a_{0}}$ unchanged, so during the short transient regime $\nu_{a}$ decreases with $I$ and then returns to almost the same $\nu_{a_{0}}$. As pressure diminishes, $\partial \zeta_{m} / \partial V$ decreases, so a larger $\Delta V$ is needed. Charging of the anode to get this larger $\Delta V$ takes longer, leaving enough time for a growth of $N_{e}$. In the meantime, $\nu_{a_{0}}$ starts decreasing as discharge voltage rises. Decreasing further the pressure, $\zeta_{m}$ is less and less capable of balancing alone a decrease of $n_{g}$, which is then compensated by a proportional decrease of $\nu_{a_{0}} \cdot\left|\partial \nu_{a_{0}} / \partial V\right|$ being decreasing, $V-p$ curve divergence initiated by the $\zeta_{m}$ saturation is extended at low pressure.

Since $\nu_{a_{0}}$ is proportional to the neutral number density at low pressure, $N_{e}=$ $I_{0}\left(e \nu_{a_{0}}\right)^{-1}$ has to increase as $1 / n_{g}$. This is confirmed by simulated data shown in figure 7 . As pressure increases, $n_{g}$ decrease is mainly compensated by an increase of $\zeta_{m}$, and $N_{e}$ decreasing rate tends to zero.

\begin{tabular}{cccc}
\cline { 2 - 3 } & & \multicolumn{2}{c}{$R_{a}(\mu \mathrm{m})$} \\
& 75 & 100 \\
\hline$R_{\text {end }}(\mathrm{mm})$ & 1.5 & - & $1130 \mathrm{~V}$ \\
& 3 & $1115 \mathrm{~V}$ & $1175 \mathrm{~V}$ \\
\hline
\end{tabular}

Table 4. Simulated discharge voltage for various anode radii $\left(R_{a}\right)$ and end plates holes radii $\left(R_{\text {end }}\right)$ for $p=3.88 .10^{-3}$ mbar.

To prevent divergence, and thus extend the optimal range of working conditions, McClure [1] proved experimentally that a solution was to decrease the anode radius or end plates holes radii. As shown in table 4, simulations confirm efficiency of both methods. We already explained how smaller wire radii yield better ionization efficiency (or similarly lower $\nu_{a_{0}}$ ) and thus lower discharge voltage. On the other hand, smaller end apertures lead to better confinement of electrons. This improved confinement allows higher electron density without voltage increase. Looking at figure 7 , this indeed means translating the point of the $V-p$ curve divergence to lower pressures.

\section{Summary}

In this paper, a 2D3v particle in cell code was used to study a wire discharge and its mode transition. Simulations reproduce fairly well the two discharge modes and their distinct properties - e.g. glow intensity and discharge voltage. Longitudinal constriction and voltage drop at the mode transition match closely the experimental data.

The simulation results justify to model the discharge near the mode transition as a unipolar ion current between cylindrical electrodes and allow us to conclude that the diffuse mode is a space charge dominated regime. A modified Child Langmuir law accounting for collisional effects is proposed and its ability to predict mode transition is successfully validated against experimental and PIC results. Discharge voltage dependence on pressure is satisfactorily explained in light of electrons ionization frequency variations.

Despite a close match in terms of relative intensity levels, glow longitudinal profiles of the diffuse mode obtained from experiments and simulations for various 
operating conditions differ slightly, experimental profiles being a little wider. Diffusion of light on the electrodes was mentioned as a plausible contributor to that profile broadening, deexcitation of the helium metastable states being possibly another one. To go further, the addition of a collisional radiative module to the existing code would permit to distinguish between the various excited states and thus allow, among others things, to discriminate the influence of metastable states on emission profiles.

\section{References}

[1] McClure G W 1963 Appl. Phys. Lett. 2 233-234

[2] Kingdon K H 1923 Phys. Rev. B 21 408-418

[3] Pigache D and Fournier G 1975 J. Vac. Sci. Technol. 12 1197-1199

[4] McIlraith A 1966 Nature 2121422

[5] Urai H, Hotta E, Maeyama M S, Yasui H and Tamagawa T 1994 Jpn. J. Appl. Phys. 33 42434246

[6] Yasui H, Tamagawa T, Ohshima I, Urai H and Hotta E 1995 Radiat. Phys. Chem 46 499-502

[7] Urai H, Kurosawa T, Okino A, Hotta E, Yasui H, Tamagawa T and Ko K C 1997 Rev. Sci. Instrum. 68 3346-3350

[8] Chalise P R, Watanabe M, Okino A, Ko K and Hotta E 2003 Plasma Sources Sci. Technol. 12 235-243

[9] Makarov M, Loumani Y and Kozyrev A 2006 J. Appl. Phys. 100033301

[10] Makarov M, Loumani Y, Minea T, Gousset G and Kozyrev A 2006 Europhys. Lett. 74 431-437

[11] Minea T M, Loumani Y, Makarov M and Gousset G 2007 Plasma Processes Polym. 4 S973-S977

[12] Pigache D 1987 Proc. of the XVIII Int. Conf. on Phenomena in Ionized Gases (Swansea) vol 4 pp 782-783

[13] Rushton G J, O'Shea K R and Fitch R K 1973 J. Phys. D: Appl. Phys. 6 1167-1172

[14] Hotta E, Suzuki M, Yasui H and Tamagawa T 1991 Proc. of the IEEE Int. Conf. on Plasma Science (Williamsburg) p 198

[15] Mohamed A, Pot T and Chanetz B 1995 Int. Congress on Instrumentation in Aerospace Simulation Facilities (Dayton) pp 14.1-14.4

[16] Francis G 1956 The glow discharge at low pressure Encyclopedia of Physics (Springer Berlin)

[17] Dawson J M 1983 Rev. Mod. Phys. 55 403-447

[18] Birdsall C and Langdon A 1985 Plasma Physics via Computer Simulation (McGraw-Hill)

[19] Birdsall C K 1991 IEEE Trans. Plasma Sci. 19 65-85

[20] Hartmann P, Donko Z, Bano G, Szalai L and Rozsa K 2000 Plasma Sources Sci. Technol. 9 183-190 ISSN 0963-0252

[21] Stone P M, Kim Y K and Desclaux J P 2002 J. Res. Nat. Inst. Stand. Technol. 107 327-337

[22] Surendra M, Graves D B and Jellum G M 1990 Phys. Rev. A 41 1112-1125

[23] Opal C B, Peterson W K and Beaty E C 1971 J. Chem. Phys. 55 4100-4106

[24] Skullerud H R 1968 J. Phys. D: Appl. Phys. 1 1567-1568

[25] Kaminsky M 1965 Atomic and Ionic Impact Phenomena on Metal Surfaces (Springer-Verlag, Berlin)

[26] Katz I, Parks D E, Mandell M J, Harvey J M, Brownell D H J, Wang S S and Rotenberg M 1977 A three dimensional dynamic study of electrostatic charging in materials Tech. Rep. NASA-CR-135256; SSS-R-77-3367

[27] Bohm C and Perrin J 1993 Rev. Sci. Instrum. 64 31-44

[28] Szapiro B, Rocca J J and Prabhuram T 1988 Appl. Phys. Lett. 53 358-360

[29] Vaughan J 1989 IEEE Trans. Electron Devices 36 1963-1967 ISSN 0018-9383

[30] Scholtz J J, Dijkkamp D and Schmitz R W A 1996 Philips J. Res. 50 375-389 ISSN 0165-5817

[31] Furman M A and Pivi M T F 2002 Phys. Rev. Spec. Top. Accel Beams 5124404

[32] Langmuir I 1913 Phys. Rev. 2 450-486

[33] Donko Z, Rzsa K, Tobin R C and Peard K A 1994 Phys. Rev. E 49 3283-3289

[34] Druyvesteyn M J and Penning F M 1940 Rev. Mod. Phys. 12 87-176

[35] Von Engel A 1954 Ionized Gases (American Inst. Phys.) 